\title{
Analysis of Eddy Currents Induced by Transverse and Longitudinal Gradient Coils in Different Tungsten Collimators Geometries for SPECT/MRI Integration
}

\author{
Amine M. Samoudi ${ }^{1 *}$, Karen Van Audenhaege ${ }^{2}$, Günter Vermeeren ${ }^{1}$, Michael Poole $^{3}$, \\ Emmeric Tanghe ${ }^{1}$, Luc Martens ${ }^{1}$, Roel Van Holen ${ }^{2}$, Wout Joseph ${ }^{1}$
}

${ }^{1}$ Department of Information Technology (INTEC), Ghent University/iMinds, Gaston crommenlaan 8 box 201, 9050 Ghent, Belgium

${ }^{2}$ Electronics and Information Systems (ELIS), Ghent University/iMinds, Campus Heymans Block B, De Pintelaan 185 , B-9000 Gent, Belgium

${ }^{3}$ Institute of Neuroscience and Medicine - 4, Forschungszentrum Jülich GmbH, 52425 Jülich, Germany

*Correspondence to: Amine M. Samoudi, ir. Department of Information Technology (INTEC), Ghent University/iMinds, Gaston crommenlaan 8 box 201, 9050 Ghent, Belgium.

E-mail: amine.samoudi@intec.ugent.be

Word account of the body of the text: 3881

Running title: Induced Eddy Currents in SPECT/MRI

Key words: MRI; SPECT; Eddy currents; Gradient coils; Collimator; Electromagnetic simulation 


\section{ABSTRACT}

Purpose: We investigated the temporal variation of the induced magnetic field due to the transverse and the longitudinal gradient coils, in tungsten collimators arranged in hexagonal and pentagonal geometries with and without gaps between the collimators.

Methods: We modeled $\mathrm{x}, \mathrm{y}$, and z-gradient coils and different arrangements of SPECT collimators using FEKO, a 3D electromagnetic simulation tool. A time analysis approach was used to generate the pulsed magnetic field gradient. The approach was validated with measurements using a 7T MRI scanner.

Results: Simulations showed an induced magnetic field representing $4.66 \%$ and $0.87 \%$ of the applied gradient field (gradient strength $=500 \mathrm{mT} / \mathrm{m}$ ) for longitudinal and transverse gradient coils, respectively. These values can be reduced by $75 \%$ by adding gaps between the collimators for the pentagonal arrangement, bringing the maximum induced magnetic field to less than $2 \%$ of the applied gradient for all the gradient coils.

Conclusion: Characterization of the maximum induced magnetic field shows that by adding gaps between the collimators for an integrated SPECT/MRI system, eddy currents can be corrected by the MRI system to avoid artifact. The numerical model was validated and was proposed as a tool for studying the effect of a SPECT collimator within the MRI gradient coils.

Key words: MRI; SPECT; Eddy currents; Gradient coils; Collimator; Electromagnetic simulation 


\section{INTRODUCTION}

Medical imaging technology is shifting from single to multi-modality imaging. Single-Photon Emission Computed Tomography (SPECT) and Magnetic Resonance Imaging (MRI) each have their respective advantages and limitations. Combining these two technologies in a synergistic manner would allow researchers to exploit the strengths of both techniques. SPECT can provide insight into a wide range of biological processes. However, its relatively poor spatial resolution can make unambiguous localization of the probes extremely difficult, especially when the images lack significant anatomical detail for reference (1). In contrast to SPECT, magnetic resonance imaging (MRI) can provide exceptionally high spatial resolution anatomical information as well as localized chemical and physical information such as metabolite concentrations and water diffusion characteristics.

Integrated whole-body Positron Emission Tomography PET/MR hybrid imaging combines excellent soft tissue contrast and various functional imaging parameters provided by MR with high sensitivity and quantification of radiotracer metabolism provided by positron emission tomography. In 2010, the first commercially available whole-body systems for PET/MR hybrid imaging entered the market, based on two separate MR and PET imagers in one room (Philips Ingenuity TF PET/MRI, Best, The Netherlands) (2), followed by a fully integrated whole-body PET/MR hybrid imaging system (Biograph mMR, Siemens AG, Healthcare Sector, Erlangen, Germany) (3) that enables simultaneous PET/MR data acquisition. Since then, the number of worldwide installations of PET/MR systems has steadily increased, making possible the introduction of new diagnostic applications and products in oncology, neurology, pediatric oncology, and cardiovascular disease (4). While the first clinical evaluation is under way (5-7), integrated PET/MR, however, is technically challenging and demands for new technologies and innovative solutions. Current research topics include: attenuation correction of human soft tissues and of hardware components have to be MRbased, the lack of bone information with MR imaging, the limited field-of-view in MR, and the implementation of motion correction technologies (4).

While combined PET and MRI is rapidly gaining popularity, no commercial systems for combining SPECT and ultrahigh filed MRI have been developed. Goetz et al. (8) used a strategy similar to PET-CT (Computed Tomography) systems in which a small animal SPECT system was brought in close proximity to a separate low field (0.1 T) MRI system for co-registered imaging. Since they performed sequential SPECT and MR imaging, their 
technique cannot be qualified as a truly integrated multimodality system with simultaneous data acquisition. Furthermore, the use of a low magnetic field is sub-optimal and obvious given the state of the art of the ultra-high field MRI system presently available. Hamamura et al. (1) developed a miniaturized dual-modality SPECT/MRI (MRSPECT) system and demonstrated the feasibility of simultaneous SPECT and MRI data acquisition. A cadmium zinc telluride (CZT) nuclear radiation detector was interfaced with a specialized radiofrequency (RF) coil and placed within a whole-body 4T MRI system. Their work can be considered as a feasibility study with simplified models to proof the possibility of simultaneous acquisition of SPECT and MRI data. Cai et al. (9) presented a stationary MR compatible small animal imaging SPECT system development and preliminary imaging performance. Their system is based on twenty second-generation energy-resolved photoncounting (ERPC) CdTe detectors. Each detector is associated with four pinholes on average. Another ongoing work for SPECT/MRI is the INSERT (Integrated SPECT/MRI for enhanced Stratification in Radio-chemo Therapy) project (10). Their final goal is to develop a custom SPECT apparatus, that can be used as an insert for commercially available MRI systems such as $3 \mathrm{~T}$ MRI with 59-cm-bore diameter. Two SPECT prototypes will be developed, one dedicated to preclinical imaging, the second one dedicated to clinical imaging. Finally, mediso (11) developed an in-line whole-body preclinical SPECT/MRI system that was firstto-market with a 1-Tesla permanent magnet, 450-mT/m gradient strength, up to $200 \times 250$ $\mathrm{mm}$ reconstructed FOV and $275-\mu \mathrm{m}$ spatial resolution for SPECT components.

While the integration of SPECT and MRI for simultaneous data acquisition offers numerous advantages and new opportunities, it also presents many technological challenges. One of them is the presence of eddy currents in the collimator due to the pulsed magnetic field gradients.

Magnetic resonance imaging techniques combine pulsed magnetic field gradients with frequency selective radio-frequency pulses to achieve spatial localization of the nuclear magnetic resonance (NMR) signal (12); in these approaches, the currents flowing in three gradient coils are rapidly switched on/off to encode the spatial position of the NMR active nuclei by a linear spatial variation of the gradient field along the $\mathrm{x}, \mathrm{y}$, and $\mathrm{z}$ coordinates. However, according to Faraday's law, any time-varying magnetic field results in an eddy current in a conducting object. Therefore, this rapid switching of gradient coils induces spatially and temporally-varying eddy currents within the conducting structures of the MRI scanner and in the collimator required for SPECT, which typically has a high conductivity. 
The undesired magnetic field produced by these eddy currents opposes and distorts the linear gradient fields in the region of interest (ROI), which results in image artifacts (13-15). Other effects concern the thermal load in the cryostat of the superconducting magnet, which may lead to increased boil-off of the cryogens (can even cause magnetic quenching in extreme cases) and acoustic noise due to their interaction with the $B_{0}$ field (16). Although many approaches have been proposed to minimize the generation of eddy currents (active and passive shielding coils (17-21), current pulse pre-emphasis $(13,15,22)$, less conductive magnet bore materials and alternative cryostat configurations (23-26)), significant distortions will often remain, mainly in the short time interval after the gradient is switched off. This is particularly the case in the presence of highly conductive objects where eddy currents are characterized by long time constants (15).

Simulating eddy currents in conducting objects is particularly computationally intensive. A variety of numerical approaches has been proposed to deal with this problem. These methods propose an efficient computer modeling tool that would reduce the experimental efforts during prototyping and development of pre-clinical SPECT/MRI or PET/MRI systems. Differential methods such as Finite Element Method (FEM) $(24,27)$ and Finite Difference Time-Domain (FDTD) were proposed and adapted to simulate eddy currents. Trakic et al (28) developed a three-dimensional FDTD method in cylindrical coordinates for the modeling of low-frequency transient eddy currents in MRI, as an extension of the work of Liu et al (29). Integral methods, such as the Boundary Element Method (BEM) (30) or the Method of Moments (MoM), only require the discretization of the active parts, neglecting the surrounding air and introducing a correct far boundary condition. Their formulations typically give rise to fully populated matrices, meaning that the storage requirements and computational time will typically grow linearly with the geometry and the required solution frequency (31). Another integral method, the network method, has been applied in the analysis of the currents induced by axially symmetric coils (e.g. z-gradient) in a realistic cryostat $(16,20,23)$, and even coupled in Fourier space to accurately evaluate the currents induced by coils of arbitrary geometry in cylindrical coordinates $(26,32)$.

The objective of this work was to use numerical models to study different designs of a tungsten collimators for pre-clinical SPECT/MRI, and the eddy currents in these collimators due to $\mathrm{x}, \mathrm{y}$, and z-gradient coils for different arrangements of tungsten collimators. With current technology, collimators can be produced by additive manufacturing (33), which gives new degrees of freedom to the design. An x, y, and z-gradient coil for pre-clinical systems 
(35) was simulated with the collimators using FEKO (31) (MoM with low frequency stabilization and a time analysis approach used to generate the pulsed magnetic field gradient). The results of the simulations are then compared to measurements using a 7T MRI scanner (Bruker Pharmascan).

The novelty of this paper is:

i. The application of a numerical model to study the specific problem of combining SPECT (collimators) with MR scanner for SPECT/MRI system.

ii. Optimization of the collimator design to reduce eddy currents' effect.

\section{THEORY}

\section{Eddy current model}

The conducting structures that support the eddy currents are approximated as inductiveresistive (LR) circuits. Mutual inductance between the gradient coil and conducting structures allows currents to be induced by the gradient coil (12). A straightforward analysis of this model approximates the eddy current as a superposition of multiple exponential decay terms as a Taylor expansion of the eddy currents (15).

According to previously described quasi-static eddy current models (13), the temporal behavior of the eddy current field $G_{e}(t)$ induced by a nominal gradient waveform $G(t)$ can be described as follows:

$$
\mathrm{G}_{\mathrm{e}}(\mathrm{t})=-\frac{\mathrm{dG}(\mathrm{t})}{\mathrm{dt}} \otimes \mathrm{H}(\mathrm{t})
$$

Where $\mathrm{H}(\mathrm{t})$ represents the eddy current impulse response function and $\mathrm{dG}(\mathrm{t}) / \mathrm{dt}$ the time derivative of $\mathrm{G}(\mathrm{t})$.

The eddy-current impulse response $\mathrm{H}(\mathrm{t})$ is given by a sum of decaying exponentials, characterized by amplitude constants $\alpha_{\mathrm{n}}$ (which depend on the inductance and the mutual inductance of the $\mathrm{n}^{\text {th }}$ coupled eddy-current mode with the gradient coil) and time constants $\tau_{n}$ (which depend on the resistance and the inductance of the $n^{\text {th }}$ coupled eddy-current mode) as follows:

$$
H(t)=u(t) \sum_{n=0}^{N-1} \alpha_{n} e^{-t / \tau_{n}}
$$


Where $u(t)$ is the unit step function, and $N$ the number of multiple exponential terms.

\section{METHODS}

\section{Simulation platform}

The gradient coils and the collimators were modeled with FEKO, a 3D full-wave electromagnetic simulation platform. FEKO uses the Method of Moments (MoM) that provides full wave solutions of Maxwell's integral equations in the frequency domain. We activated the low-frequency stabilization and we used the Volume Equivalence Principle (VEP) for meshing the collimator. The VEP allowed the creation of dielectric bodies from cuboids or tetrahedral, and it is advantageous for low frequencies simulations (31).

FEKO provided time analysis functionality and then electromagnetic problems can be analyzed in the time domain. The relevant computations were performed in the frequency domain and Fast Fourier Transform (FFT) algorithms were used to transform the data to the time domain. First, we extracted frequency components of the gradient pulse by applying the FFT. Then we performed a broadband simulation covering the frequency range of the gradient shape. The output was then post-processed with FEKO time domain analysis to extract the time response of the system, based on the gradient pulse.

\section{Validation}

\section{Measurements}

To validate the simulations, we performed measurements on a $7 \mathrm{~T}$ preclinical MRI from Bruker with a volume coil (Part No. T1123V3) and a rat brain surface receiver coil (Part No. T11425V3). We measured eddy currents due to a $25 \times 10 \times 40 \mathrm{~mm}^{3}$ block of lead $(\mathrm{Pb})$ with a resistivity of $186.43 \mathrm{n} \Omega . \mathrm{m}$. The block was inserted in the MR bore (at position $\mathrm{x}=20.5, \mathrm{y}=0$, and $\mathrm{z}=2 \mathrm{~mm}$, on top of the receiver coil) together with a spherical phantom with an inner diameter of $12.24 \mathrm{~mm}$, filled with $\mathrm{CuSO}_{4}(1 \mathrm{~g} / \mathrm{L})$ in $\mathrm{H}_{2} \mathrm{O}(\mathrm{CAS} \mathrm{nr}$ : 7758-99-8) with an electrical conductivity of $0.057 \mathrm{~S} / \mathrm{m}$. Measurements were performed with the phantom positioned at different locations along the $\mathrm{z}$-axis $(\mathrm{x}=0.35$ and $\mathrm{y}=0.23 \mathrm{~mm})$ and with the sequence of Fig. 1. We applied a z-gradient with a peak value of $119.35 \mathrm{mT} / \mathrm{m}$ (applied for $500 \mathrm{~ms}$ ) and a sinusoidal ramp up and down (both with duration of $248 \mu \mathrm{s}$ ). Immediately after 
the ramp-down, we acquired the free induction decay (FID) signal, whose phase relates to the magnetization as follows (4):

$$
\phi(t)=\gamma \int_{0}^{t} B_{e}\left(t^{\prime}\right) d t^{\prime}+\phi_{0}
$$

With $\phi(\mathrm{t})$ the phase of the FID, $\gamma$ the gyromagnetic ratio $\left(267.513 \mathrm{rad} / \mathrm{s} / \mathrm{T}\right.$ for protons), $\mathrm{B}_{\mathrm{e}}$ the magnetic field induced by the eddy currents in the lead block and $\phi_{0}$ a constant phase offset due to main field inhomogeneities. To cancel out the constant offset, we acquire the FID after both a positive and a negative gradient:

$$
\phi_{\mathrm{T}}(\mathrm{t})=\frac{\phi_{+}(\mathrm{t})-\phi_{-}(\mathrm{t})}{2}=\gamma \int_{0}^{\mathrm{t}} \mathrm{B}_{\mathrm{e}}\left(\mathrm{t}^{\prime}\right) \mathrm{dt}^{\prime}
$$

The magnetic field due to eddy currents was then retrieved by differentiating the phase:

$$
\mathrm{B}_{\mathrm{e}}(\mathrm{t})=\frac{1}{\gamma} \frac{\mathrm{d} \phi_{\mathrm{T}}(\mathrm{t})}{\mathrm{dt}}
$$

We performed these measurements both with and without the lead block insert and for different locations of the phantom. We did not modify the pre-emphasis settings of the MRI system.

\section{Simulations}

To validate simulations with the configuration of measurements, we considered a z-gradient coil, a representation of the phantom and the radio-frequency (RF) coil and a cuboid representing the lead block $(\mathrm{Pb})$. The phantom was modeled as a sphere of diameter $12.24 \mathrm{~mm}$ with electrical conductivity of the $\mathrm{CuSO}_{4}-5 \mathrm{H}_{2} \mathrm{O}, \sigma=0.057 \mathrm{~S} / \mathrm{m}$. The RF coil was modeled as a half cylinder (central axis $=\mathrm{z}$-axis, diameter $=37.5 \mathrm{~mm}$, height $=80 \mathrm{~mm}$, thickness $=5 \mathrm{~mm}$ ) with a perfect electric conductor (PEC) medium (a zero-resistance conductor). A cuboid of dimensions $25 \times 10 \times 40 \mathrm{~mm}^{3}$ and electrical resistivity $\rho=186.43 \mathrm{n} \Omega . \mathrm{m}$, represented the $\mathrm{Pb}$ block. The simulated gradient ramp has a sinusoidal shape with a ramp-down time of $248 \mu$ s and a gradient strength $G=119.35 \mathrm{mT} / \mathrm{m}$ to match the gradient applied in measurements. Figure 2 shows a representation of the described example. To determine the magnetic field due to eddy currents from the simulations, we first extracted the z-component of the magnetic induction B from both simulations (with and without the Pb block), then we compared the two components to quantify the added magnetic field due to the $\mathrm{Pb}$ insertion. 


\section{Numerical model}

\section{Gradient coils}

The system under investigation consisted of an $\mathrm{x}, \mathrm{y}$, and $\mathrm{z}$-gradient coils for pre-clinical systems and a SPECT collimator made of tungsten. Figure 3 shows the gradient coils, while Table 1 lists its properties and configurations. The setup was simulated with a broadband simulation from 0 to $10 \mathrm{kHz}$ with a step of $400 \mathrm{~Hz}$ to cover the frequency range of a sinusoidal ramp from $500 \mathrm{mT} / \mathrm{m}$ to $0 \mathrm{mT} / \mathrm{m}$ within $0.25 \mathrm{~ms}$. The magnetic field due to eddy currents was calculated by subtracting the z-component of the magnetic induction of the gradient coils without collimator $\left(\mathrm{B}_{\mathrm{z}}\right)$ from the $\mathrm{z}$-component of the magnetic induction of the gradient coils with the collimator $\left(\mathrm{B}_{\mathrm{z}}^{\mathrm{col}}\right)$. We then calculated the maximum value within the three-cm FOV.

We performed simulations using the sinusoidal gradient ramp with a ramp-down time of $0.25 \mathrm{~ms}$ to characterize the induced magnetic field as a function of time.

\section{Collimator}

We simulated a full-ring multi-pinhole collimator which is a part of a new, more compact microSPECT system that is under development. Deprez et al. (33) designed and produced the collimator with 20 loftholes (41) with 500- $\mu$ m-diameter pinhole openings. The density of the collimator was equal to $17.31 \pm 0.10 \mathrm{~g} / \mathrm{cm}^{3}$ (89.92 $\pm 0.05 \%$ of pure tungsten). The designed multi-lofthole collimator (16 mm thick and $70 \times 52 \mathrm{~mm}^{2}$ transverse size) was sent to the additive manufacturing company (Layerwise, Belgium) to be produced (Fig. 4 (f)). The collimator was built using direct additive manufacturing technique based on selective laser melting of high density tungsten powder. Van Holen et al (34) used this collimator in a pentagonal and hexagonal arrangement to determine the optimal microSPECT system. Results show that the authors can successfully reconstruct a Defrise disk phantom of 24-mmdiameter without any rotating system components or translation of the object. Reconstructed spatial resolution is approximately $800 \mu \mathrm{m}$ while the peak sensitivity is $0.23 \%$.

Due to the additive manufacturing process, the collimator has a different resistivity along the transversal $\left(\rho_{t}=292 \mathrm{n} \Omega . \mathrm{m}\right)$ and the longitudinal $\left(\rho_{\mathrm{l}}=108 \mathrm{n} \Omega . \mathrm{m}\right)$ direction. Since eddy currents increase with the material conductivity, we performed our simulations with a collimator of $\rho=108 \mathrm{n} \Omega$. m to cover the strongest eddy currents. 
In this paper, we performed three sets of simulations using different arrangements of the collimators. First, we used the pentagonal arrangement with $\mathrm{x}, \mathrm{y}$, and $\mathrm{z}$ gradient coils. Second, we simulated with the hexagonal arrangement and third, we investigated the influence of the gap between the collimators in the pentagonal geometry to study the effect of the geometry on the induced magnetic field (Fig. 4). Motivations for using hexagonal or even heptagonal geometry are that small animals fit better with these geometries compared to the pentagonal arrangement in the SIMRET (SImultaneous Magnetic Resonance imaging and Emission Tomography) project.

\section{RESULTS}

\section{Measurements results and validation of the simulations}

Measurements were performed after turning off the gradient for $248 \mu$ s. Figure 5 (a) shows the average value (over a sphere of radius $1 \mathrm{~mm}$, centered at $(0.345,0.23,2.8) \mathrm{mm}$ ) of the magnetic field due to eddy currents for both simulations and measurements. Figure 5 (b) shows the difference between measurements and simulations compared to the applied gradient field. The maximum variation between measurement and simulation was less than $1 \%$ of the applied gradient field.

\section{Temporal variation of the induced magnetic field for pentagonal arrangement}

Figure 6 shows the magnetic induction due to eddy currents $\left(B_{e}\right)$ as a percentage of the applied gradient field for longitudinal and transverse gradient coils. The maximum value of the induced magnetic field is $4.66 \%$ and $0.87 \%$ of the applied gradient field (gradient strength $=500 \mathrm{mT} / \mathrm{m}$ ) for longitudinal and transverse gradient coils, respectively. The applied gradient field is determined at the point in space where the eddy current is being calculated. The transverse coils induce thus less eddy currents than the longitudinal.

\section{Comparison with hexagonal and pentagonal arrangement with gaps}

In this part we investigated different arrangements of the collimators using pentagonal geometry with gaps and the hexagonal geometry for the transverse and the longitudinal 
gradient coils. Table 2 shows the maximum value of $B_{e}$ (always as a percentage of the applied gradient field) for the three configurations.

The hexagonal geometry induces larger eddy currents, and by adding relatively small gaps between the collimators $(1.7 \mathrm{~mm})$, the maximum value of the induced magnetic field by $50.6 \%$ and $75.8 \%$ for transverse and longitudinal gradient coils, respectively, bringing back the maximum value of the induced field to less than $2 \%$ of the applied gradient field.

\section{DISCUSSION}

Figures 5 (a) and 5 (b) show that the model used in simulations is coherent with measurements for the lead block. Simulations with pentagonal arrangements of collimators show that we should expect a maximum value of induced magnetic field representing $4.66 \%$ and $0.87 \%$ for longitudinal and transverse gradient coils, respectively. The transverse coils induce thus less eddy currents than the longitudinal ones, due to the wire distribution for the two types of the coils and the collimators' position inside the coils. In fact, since gradient fields are along the $\mathrm{z}$ direction for both of the coils and increase linearly with the same ratio $\left(G_{x}=G_{y}=G_{z}\right)$, the collimator, by its geometry and position, is more exposed to the gradient fields in the longitudinal configuration (Figure 7).

Regarding our SIMRET project; if the eddy currents induce a magnetic field that is larger than $2 \%$ of the gradient field, artifacts will arise. This guideline value of $2 \%$ is experimentally derived during measurements for the SIMRET project. To reduce the percentage of the induced eddy currents under this threshold, we investigated the hexagonal geometry and we added gaps between the collimators.

The hexagonal system has a higher sensitivity than the pentagonal one (34) but it induces more eddy currents for both types of gradient coils. This is due to the increase in number of the collimators: 6 instead of 5 for the pentagonal arrangement; because we have more conductive volume in the hexagonal arrangement.

Adding gaps between the collimators reduces the maximum value of $\mathrm{B}_{\mathrm{e}}$ by $75 \%$ and brings it to less than $2 \%$ for all the gradient coils. However, the individual pieces should be electrically isolated; otherwise, the values of $1.13 \%$ and $0.43 \%$ should be revised to include eventual eddy current in the connections between the collimators. 
Figure 6 shows that the magnetic field produced by the eddy current changes sign. These relatively small values (the maximum negative value represents $2.71 \%$ of the maximum positive value) can be justified by two facts: first, this might be due to competing eddy currents on different surfaces with slightly different time constants. Second, this can be due to the numerical uncertainties. Bakker et al (38), gives at least $9 \%$ margin of error due to the numerical simulation.

Results show that we still have residual eddy currents that induce a spatiotemporal magnetic field, which can be further reduced nowadays using two standard practices. First, preemphasis techniques use eddy current models and constants to temporally modify the gradient pulse, in a way that the combination of the nominal gradient field and the induced field produce the desired pulse shape (22). Second, active shielding, in which a secondary coil surrounding the first is introduced. The wires in this second screening coil are positioned so as to cancel the field from the inner coil in the region outside the screen $(20,21)$. Both approaches are often used in combination in most MRI scanners. Poole et al. (36) proposed an eigenmode analysis of eddy currents to reduce the complex spatiotemporal eddy current field variation to a purely temporal variation. In this way, pre-emphasis would work exactly over the whole region of interest with a single time constant filter since the spatial form of the eddy current field stays exactly the same.

We assumed a linearity of the system in the method of extraction of the induced magnetic field due to eddy currents. Gradient coils and the collimator are a passive structures simulated with electromagnetic tools using linear Maxwell's equations. We note here that we have focused only on the eddy current induced in the tungsten collimators. The proposed work does not consider the effect of coupling of the gradient and/or RF coils, nor the eddy currents on the cryostat magnet, which leads to some remnant errors. However, these could be accounted for through extensions of the methodology presented here, with a simple extension of the numerical model. Different works in the literature investigated eddy currents in the cryostat $((14,24,27,28)$ for example) or the RF coils $(37)$.

The simulation results demonstrate that the approach could be effectively used to investigate eddy current problems in conductive materials. 


\section{CONCLUSION}

A numerical model of the $\mathrm{x}, \mathrm{y}$, and z-gradient coils with different arrangements of the collimators was studied to investigate eddy currents in the tungsten collimators due to the gradient fields for SPECT/MRI system. Simulations were performed using a 3D electromagnetic simulator with a time analysis tool. We investigated the induced magnetic field and compared it to the applied gradient for pentagonal and hexagonal arrangement of the collimators. We also studied the effect of adding gaps between the collimators in the pentagonal geometry to reduce eddy current density under $2 \%$ of the applied gradient field. The numerical model was validated with measurements and it was proposed as an efficient tool for studying the effect of SPECT collimators within the MRI gradient coils. Future research could consist of further segmentation of the collimator into an arrangement of smaller isolated conductors without reducing the quality of the SPECT images. This would reduce the extent of the contiguous eddy currents and likely further decrease artifacts in the MR images. Validation of the simulations with anisotropic material could also be part of future studies.

\section{ACKNOWLEDGMENTS}

Grant sponsor: This work was supported by the iMinds SIMRET ('SImultaneous Magnetic Resonance imaging and Emission Tomography') project, co-funded by iMinds, a research institute founded by the Flemish Government in 2004, and the involved companies and institutions.

We would like to thank Maarten Cauwe from the Centre for Microsystems Technology (CMST) for his help with the resistance measurements.

Roel Van Holen is funded by the Research Foundation-Flanders (FWO, Belgium) and by the Ghent University.

Karen Van Audenhaege is funded by the agency for Innovation by Science and Technology Flanders (IWT, Belgium). 
Michael Poole is a grateful recipient of a Marie Curie international outgoing Fellowship of the European Community's Seventh Framework Program, the project number FP7-PEOPLEIIF-331751 : MCODE

\section{REFERENCES}

1. Hamamura M, Ha S, Roeck W, Muftuler T, Wagenaar D, Meier D, Patt B, Nalcioglu O, Development of an MR-compatible SPECT system (MRSPECT) for simultaneous data acquisition, Phys. Med. Biol., vol. 2010, no. 55, pp. 1563-1575, 2010. Phys. Med. Biol. 2010:55:1563-1575.

2. Zaidi H, Ojha N, Morich M, et al. Design and performance evaluation of a whole-body Ingenuity TF PET-MRI system. Phys Med. Biol 2011;21:3091-3106.

3. Delso G, Furst S, Jakoby B, et al. Performance measurements of the Siemens mMR integrated whole-body PET/MR scanner. J Nucl Med 2011;52:1914-1922.

4. Quick H, Integrated PET/MR, J. Magn. Reson. Imag. 2014:39:243-258

5. Drzezga A, Souvatzoglou M, Eiber M, et al. First clinical experience with integrated whole-body PET/MR: comparison to PET/CT in patients with oncologic diagnoses. J Nucl Med 2012;53:845855.

6. Schwenzer NF, Schraml C, Muller M, et al. Pulmonary lesion assessment: comparison of wholebody hybrid MR/PET and PET/CT imaging-pilot study. Radiology 2012;264:551-558.

7. Quick H, von Gall C, Zeilinger M, et al. Integrated whole-body PET/MR hybrid imaging: clinical experience. Invest Radiol 2013;48:280-289.

8. Goetz C, Breton E, Choquet P, Israel-Jost V, Constantinesco A, SPECT Low-Field MRI System for Small-Animal Imaging, J Nucl Med 2008;49:88-93.

9. Cai L, Lai X, Shen Z, Chen CT, Meng LJ, MRC-SPECT: A sub-500 mm resolution MRcompatible SPECT system for simultaneous dual-modality study of small animals, Nuclear Instruments and Methods in Physics Research A 2014;734:147-151.

10. Busca P, et al. Simulation of the expected performance of INSERT: A new multi-modality SPECT/MRI system for preclinical and clinical imaging. Nucl Instrum and Methods in Physics Research A 2014;734:141-146.

11. Mediso Medical Imaging Systems. Available online at www.mediso.com; visited on June 2014.

12. Bernstein MA, King KF, Zhou X, Handbook of MRI pulse sequences, Boston: Academic Press 2004.

13. Morich MA, Lampman DA, Dannels WR, Handler, Goldie FTD, Exact temporal eddy current compensation in magnetic resonance imaging systems, IEEE, Medical Imaging 1988;7:247-254. 
14. Crozier S, Eccles C, Beckey F, Fields J, Doddrell D, Correction of eddy current-induced B0 shifts by receiver reference phase modulation, J of Magn Reson. 1988;7:247-254.

15. Jehenson P, Westphal M, Schu N, Analytical method for the compensation of eddy-current effects induced by pulsed magnetic field gradients in NMR systems, J of Magn Reson. 1990;90:264-278.

16. Edelstein WA, Kidane TK, Varacila V, Baig T, Eagan T, Cheng YCN, Brown RW, Mallick JA, Active-passive gradient shielding for MRI acoustic noise reduction, Magn. Reson. Med.2005;53:1013-1017.

17. Mansfield P, Chapman B, Active magnetic screening of gradient coils in NMR imaging, J Magn Reson. 1986;66:573-576.

18. Turner R, Bowley RM, Passive screening of switched magnetic field gradients, J. Phys. E Sci. Instrum. 1986;19:876-879.

19. Lopez HS, Poole M, S. Crozier S, Evaluating passively shielded gradient coil configurations for optimal eddy current compensation, J. Phys. D: Appl. Phys. 2010;43:1-12.

20. Kidane T, Edelstein WA, Eagan T, Taracila V, Baig TN, Cheng YCN, Brown RW, Activepassive shielding for MRI acoustic noise reduction: network analysis, IEEE Trans Magn. 2006 ;42:3854-3860.

21. Bowtell R, Chapman B, Gradient coil design using active magnetic screening, Magn Reson Med. 1991;17:15-21.

22. Glover GH, Pelc NJ, Method for magnetic field gradient eddy current compensation. US patent No 4,698,591 (Milwaukee, WI, General Electric Company)

23. Takahashi $\mathrm{T}$, Numerical analysis of eddy current problems involving $\mathrm{z}$ gradient coils in superconducting MRI magnets, IEEE Trans Magn. 1990;26:893-896.

24. Takahashi T, Numerical analysis of eddy current problems involving saddle shaped coils in superconducting MRI magnets, IEEE Trans Magn 1991;27:3996-3999.

25. Heid O, Magnetic resonance apparatus with compensation of fields arising due to eddy currents. US patent No 6,844,733 (Munich, Siemens Aktiengesellschaft).

26. Poole M, Lopez HS, Ozaki O, Kitaguchi H, Nakajima I, Urayama S, Sato K, Fukuyama H, Crozier S, Simulation of gradient-coil-induced eddy currents and their effects on a head only HTS MRI magnet, IEEE, Trans Applied Superconductivity 2011;21:3592-3598.

27. Nabi MU, Kulkarni SV, Sule VR, Novel modeling and solution approach for repeated finiteelement analysis of eddy current systems, IEEE Trans Magn. 2004;40:21-28.

28. Trakic A, Wang H, Liu F, Lopez HS, Crozier S, Analysis of transient eddy currents in MRI using a cylindrical FDTD method, IEEE Trans Appl Supercond. 2006;16:1924-1936.

29. Liu F, Crozier S, An FDTD model for calculation of gradient induced eddy currents in MRI system, IEEE Trans Appl Supercond. 2004;14:1983-1989.

30. Canova A, Gruosso G, Repetto M, Integral methods for analysis and design of low-frequency conductive shields, IEEE Trans Magn. 2003;39:2009-17. 
31. FEKO, EM Software \& Systems-S.A. (Pty) Ltd. Available online at www.feko.info; visited on August 2014.

32. Lopez HS, Poole M, Crozier S, Eddy current simulation in thick cylinders of finite length induced by coils of arbitrary geometry, J. Magn. Reson. 2010;207:251-261.

33. Deprez K, Vandenberghe S, Audenhaege KV, Vaerenbergh JV, Holen RV, Rapid additive manufacturing of MR compatible multipinhole collimators with selective laser melting of tungsten powder, Med Phys. 2013;40.

34. Van Holen R, Vandeghinste B, Deprez K, Vandenberghe S, Design and performance of a compact and stationary microSPECT system, Med. Phys. 2013;40:112501-11

35. Poole M, Bowtell R, Novel gradient coils designed using a boundary element method, Concepts in Magn Reson. 2007;B31:162-175.

36. Poole M, Lopez HS, Crozier S, Eigenmode analysis of eddy currents and eigenmode coil design, ISMRM 2010;18:3933.

37. Zheng H, Zhao T, Qian Y, Ibrahim T, Boada F, Parallel transmission RF pulse design for eddy current correction at ultra-high field, J Magn Reson. 2012;221:139-46.

38. 38. Bakker JF, Paulides MM, Neufeld E, Christ A, Chen XL, Kuster N, van Rhoon GC, Children and adults exposed to low-frequency magnetic fields at the ICNIRP reference levels: theoretical assessment of the induced electric fields, Phys. Med. Biol. 2012;57:1815-1829

39. D. Kurup, W. Joseph, G. Vermeeren and L. Martens, Path loss model for in-body communication in homogeneous human muscle tissue, Electronics Letters, 2009;45:453-454

40. L. Roelens, W. Joseph, E. Reusens, G. Vermeeren, and L. Martens, Characterization of Scattering Parameters Near a Flat Phantom for Wireless Body Area Networks, IEEE Trans. on electromagnetic compatibility 2008;50:185-193

41. K. Deprez, R. Van Holen, and S. Vandenberghe, The lofthole: A novel shaped pinhole geometry for optimal detector usage without multiplexing and without additional shielding. Proceedings of the Nuclear Science Symposium and Medical Imaging Conference (NSS/MIC), 2011 (IEEE, Valencia, Spain), p p . 3317-3322. 
Figure 1: Pulse sequence used for the measurement of eddy currents. $G$ is the gradient strength and $\tau$ the ramp-down time. Induced magnetic field is taken after completely switching off the gradient field.

Figure 2: Experimental setup used to validate simulations. Gradient system consists of shielded z-gradient coil. The phantom is modeled by a sphere of diameter $12.24 \mathrm{~mm}$. The RF coil is modeled as a half cylinder (central axis $=$ z-axis, diameter $=37.5 \mathrm{~mm}$, height $=80 \mathrm{~mm}$, thickness $=5 \mathrm{~mm}$ ). A cuboid of dimensions $25 \times 10 \times 40 \mathrm{~mm}^{3}$ represents the $\mathrm{Pb}$ block.

Figure 3: Wire patterns for (a) X-gradient coil. (b) Y-gradient coil and(c) Z-gradient coil. (d) Pentagonal ring of the collimators centered inside the z-gradient coil. Red and blue colors are used to indicate wires in which there is a different sense of current flow.

Figure 4: The simulated collimators system geometry definition. In (a) and (c), the pentagonal and hexagonal arrangements are shown, respectively (dimensions are in $\mathrm{mm}$ ). In (b) and (d), a rendering of the respective SPECT systems is shown. Centrally, the multipinhole collimators are shown, followed by the scintillator, PMTs, and electronics. (e) Shows the pentagonal geometry with gap of $1.7 \mathrm{~mm}$ between the collimators. (f) The design of the collimator (33).

Figure 5: Validation of the simulation using the $\mathrm{Pb}$ block (a) Measured and simulated magnetic induction due to eddy currents $B_{e}$. The phantom is positioned at $(0.345,0.23$, 2.8) $\mathrm{mm}$ (b) Difference between measured and simulated induced field due to $\mathrm{Pb}$ insertion, compared to the applied gradient field. $\mathrm{B}_{\mathrm{s}}, \mathrm{B}_{\mathrm{m}}$, and $\mathrm{B}_{\mathrm{G}}$ stands for simulated, measured and applied magnetic induction, respectively.

Figure 6: $\mathrm{B}_{\mathrm{e}}$ in $\%$ of the applied gradient field of $500 \mathrm{mT} / \mathrm{m}$ for both the longitudinal and the transverse gradient coils in a FOV of $3 \mathrm{~cm}$, after switching off the gradient field.

Figure 7: Representation of the gradient field strength for:(a) X-gradient coil (transverse gradient coil) (b) Z-gradient coil (longitudinal gradient coil). The norm and the direction of the vectors represent the field's strength and sign, respectively. 


\begin{tabular}{l|l|l|l|l|l|l|l|} 
& $\begin{array}{c}\text { Inner } \\
\text { diameter } \\
(\mathbf{m m})\end{array}$ & $\begin{array}{c}\text { Outer } \\
\text { diameter } \\
(\mathbf{m m})\end{array}$ & $\begin{array}{c}\text { Length } \\
(\mathbf{m m})\end{array}$ & $\begin{array}{c}\text { Gradient } \\
\text { strength } \\
(\mathbf{m T} / \mathbf{m})\end{array}$ & $\begin{array}{c}\text { Gradient } \\
\text { efficiency } \\
(\mathbf{m T} / \mathbf{m} / \mathbf{A})\end{array}$ & $\begin{array}{c}\text { Applied } \\
\text { current } \\
(\mathbf{A})\end{array}$ & $\begin{array}{c}\text { DSV of } \\
\mathbf{3 c m}\end{array}$ \\
\hline $\mathrm{X}$ coil & 100 & 126 & 293 & 500 & 2.99 & 167.24 & $2.06 \%$ \\
\hline Y coil & 100 & 126 & 293 & 500 & 2.99 & 167.24 & $2.06 \%$ \\
\hline $\mathrm{Z}$ coil & 100 & 128 & 232 & 500 & 3.08 & 162.33 & $0.86 \%$ \\
\hline
\end{tabular}

Table 1: Parameters of the transverse and the longitudinal gradient coils.

\begin{tabular}{l|l|l|l|} 
& \multicolumn{1}{|c|}{ Pentagon } & \multicolumn{1}{c|}{$\begin{array}{c}\text { Pentagon } \\
\text { with gaps }\end{array}$} & \multicolumn{1}{c|}{ Hexagon } \\
\hline Transverse & $0.87 \%$ & $0.43 \%$ & $0.97 \%$ \\
\hline Longitudinal & $4.66 \%$ & $1.13 \%$ & $6.17 \%$ \\
\hline
\end{tabular}

Table 2: The maximum induced magnetic field as a $\%$ of the applied gradient field $(\mathrm{G}=500 \mathrm{mT} / \mathrm{m}, \tau=0.25 \mathrm{~ms})$ in a FOV of $3 \mathrm{~cm}$. 


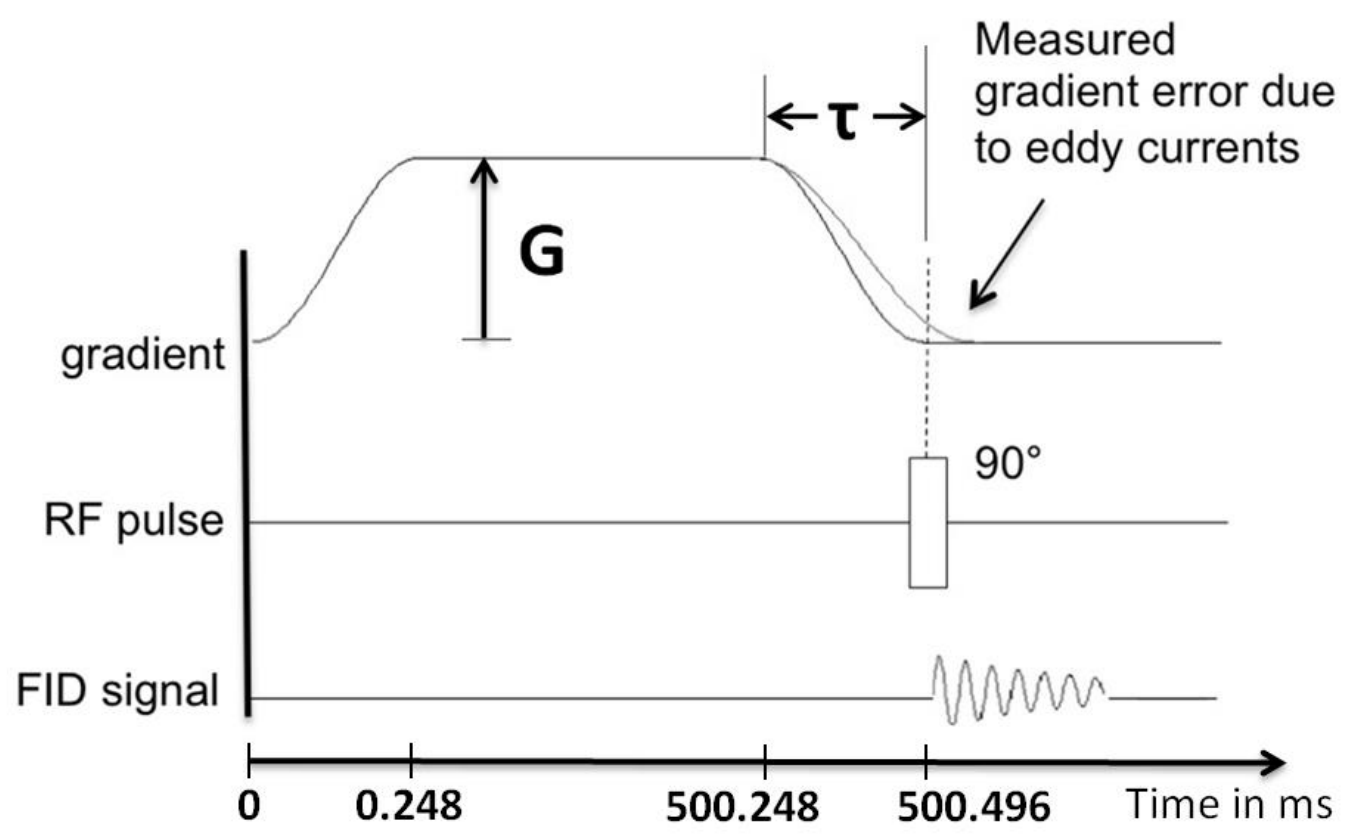

Figure 1
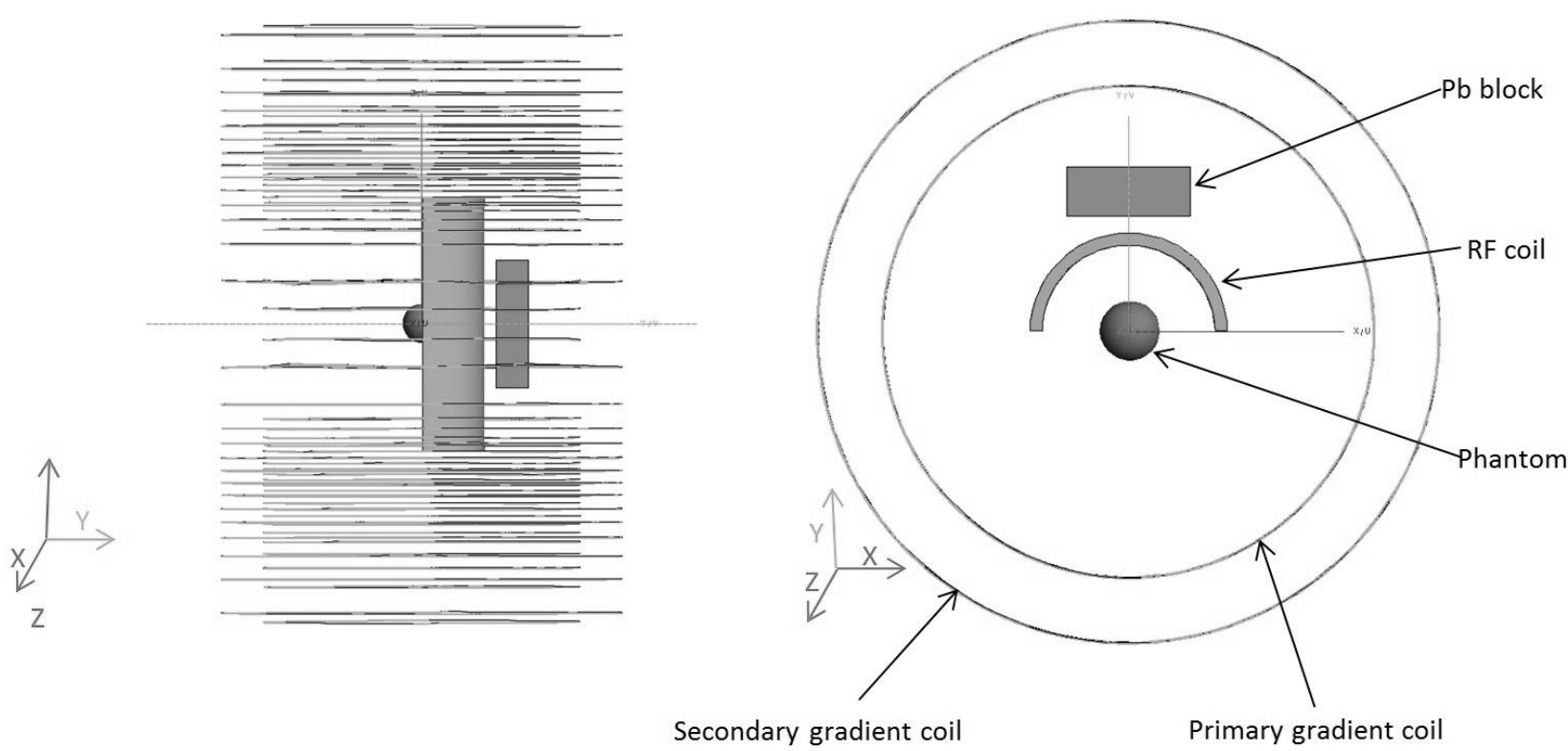

Figure 2 


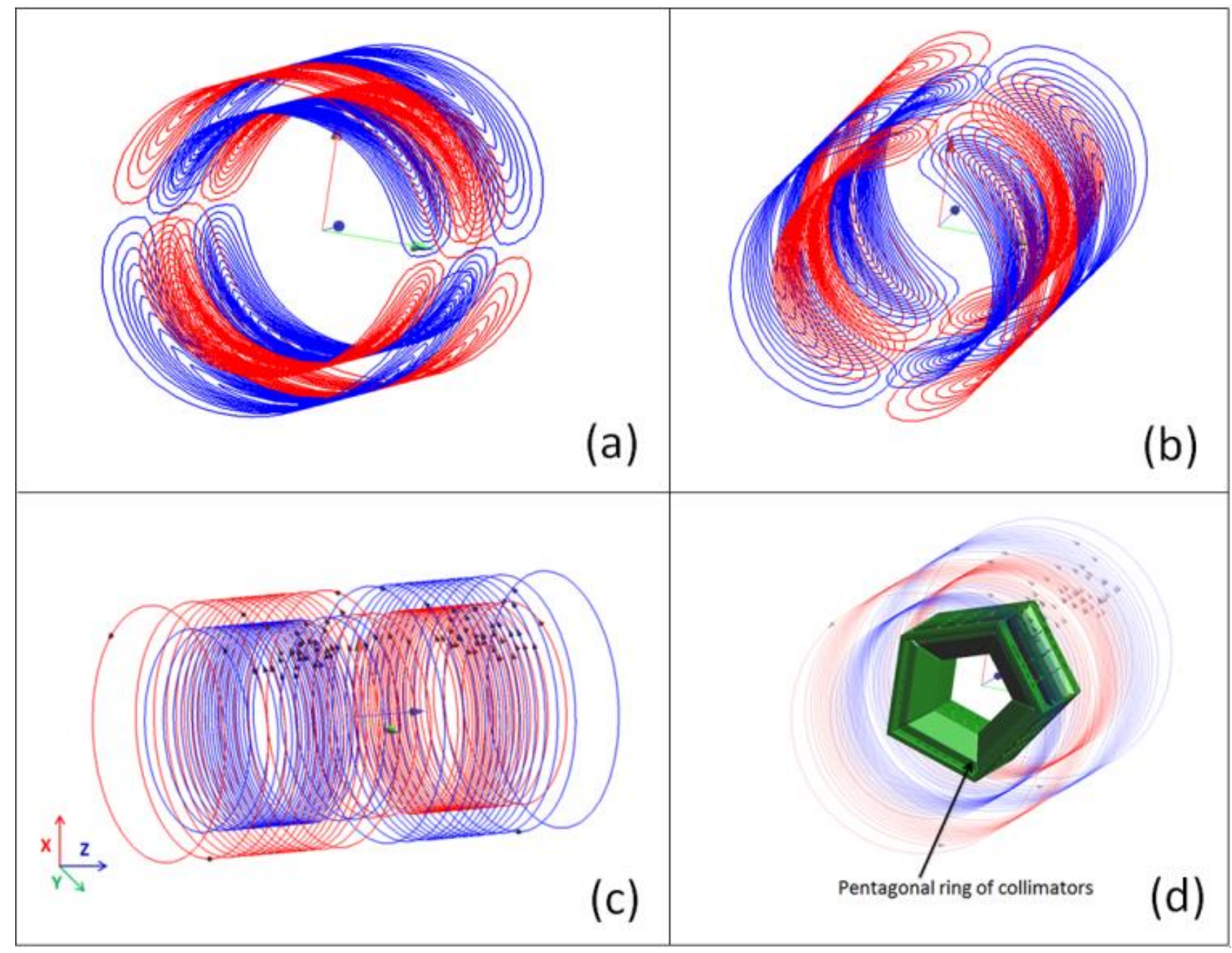

Figure 3 


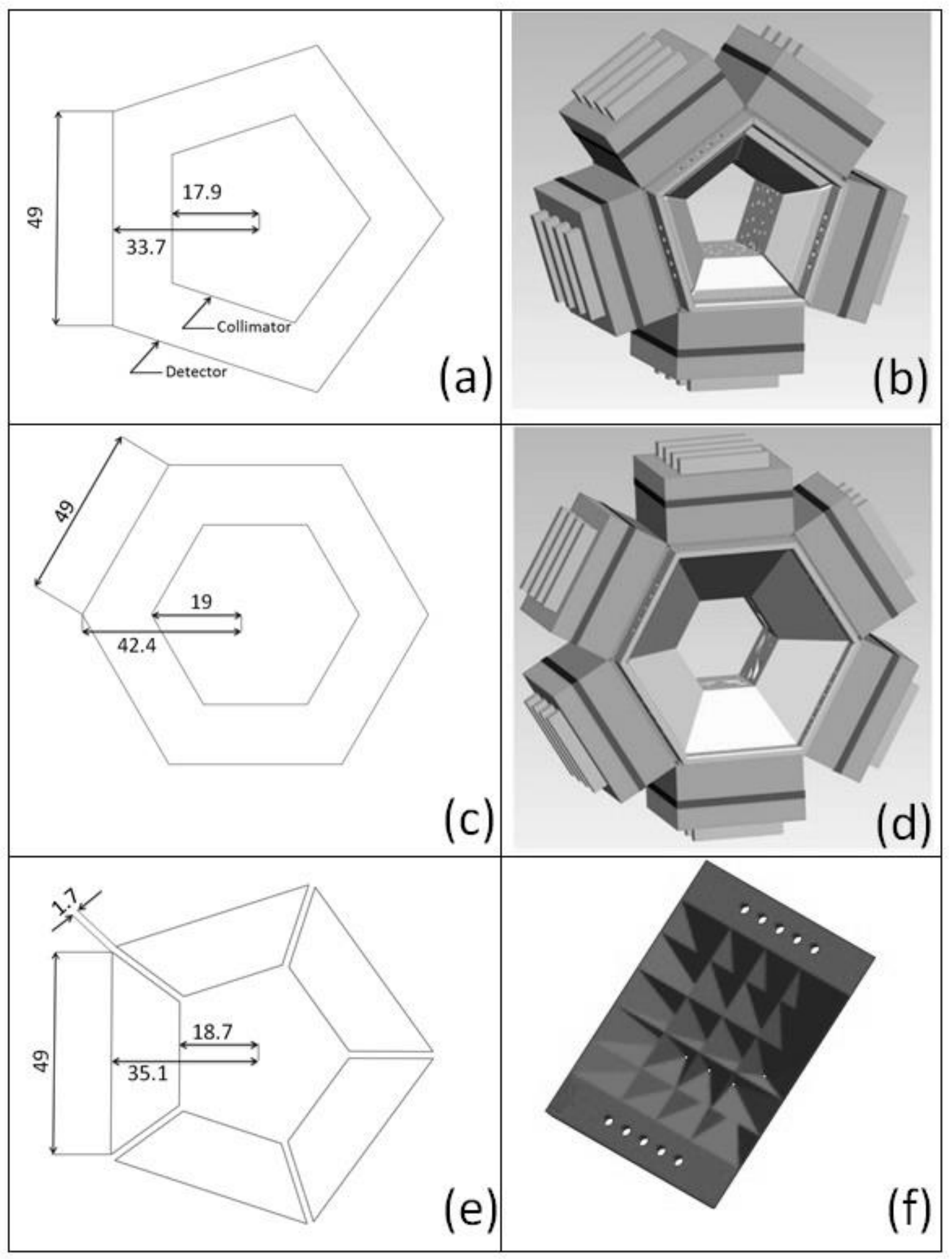

Figure 4 


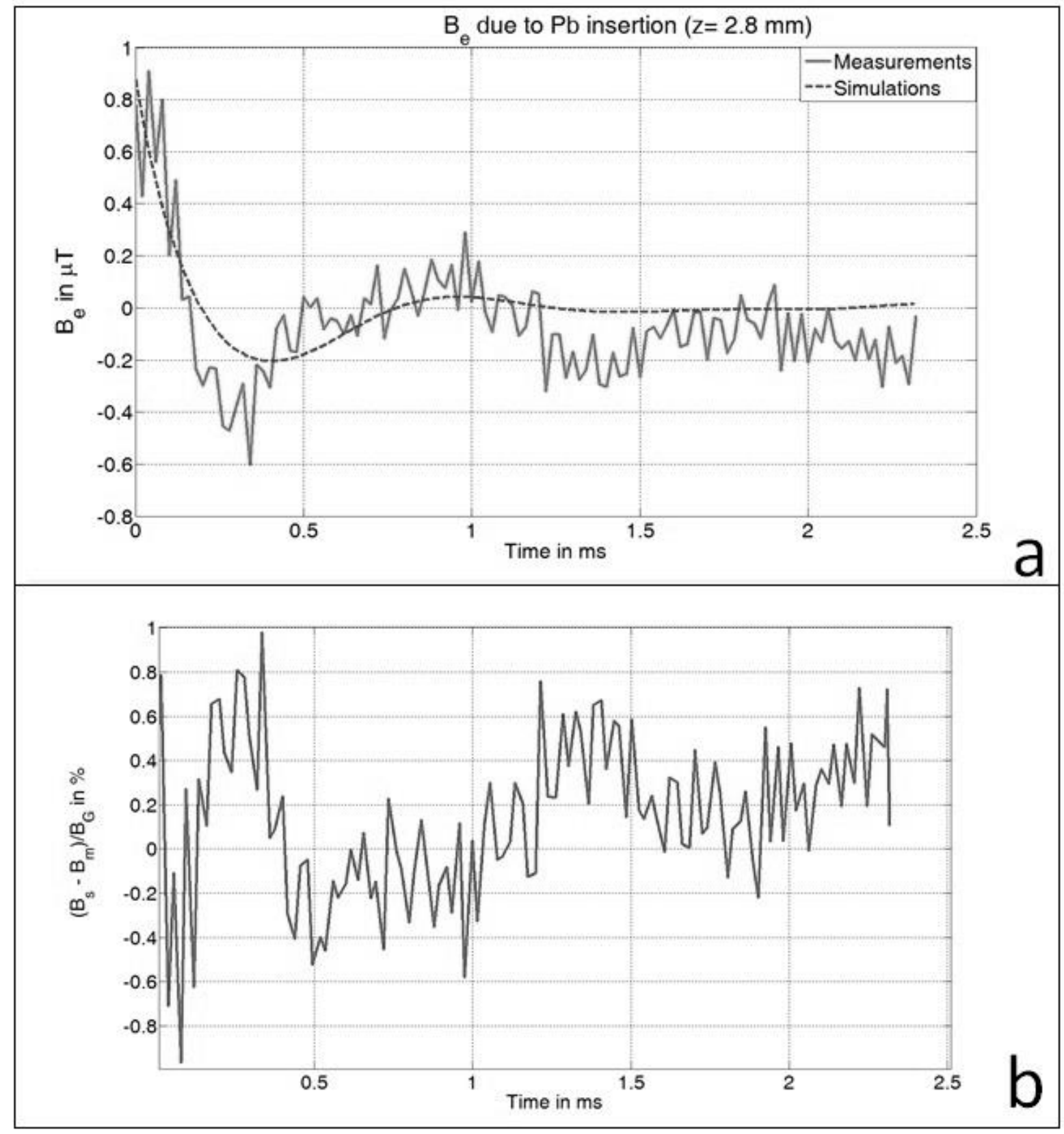

Figure 5 


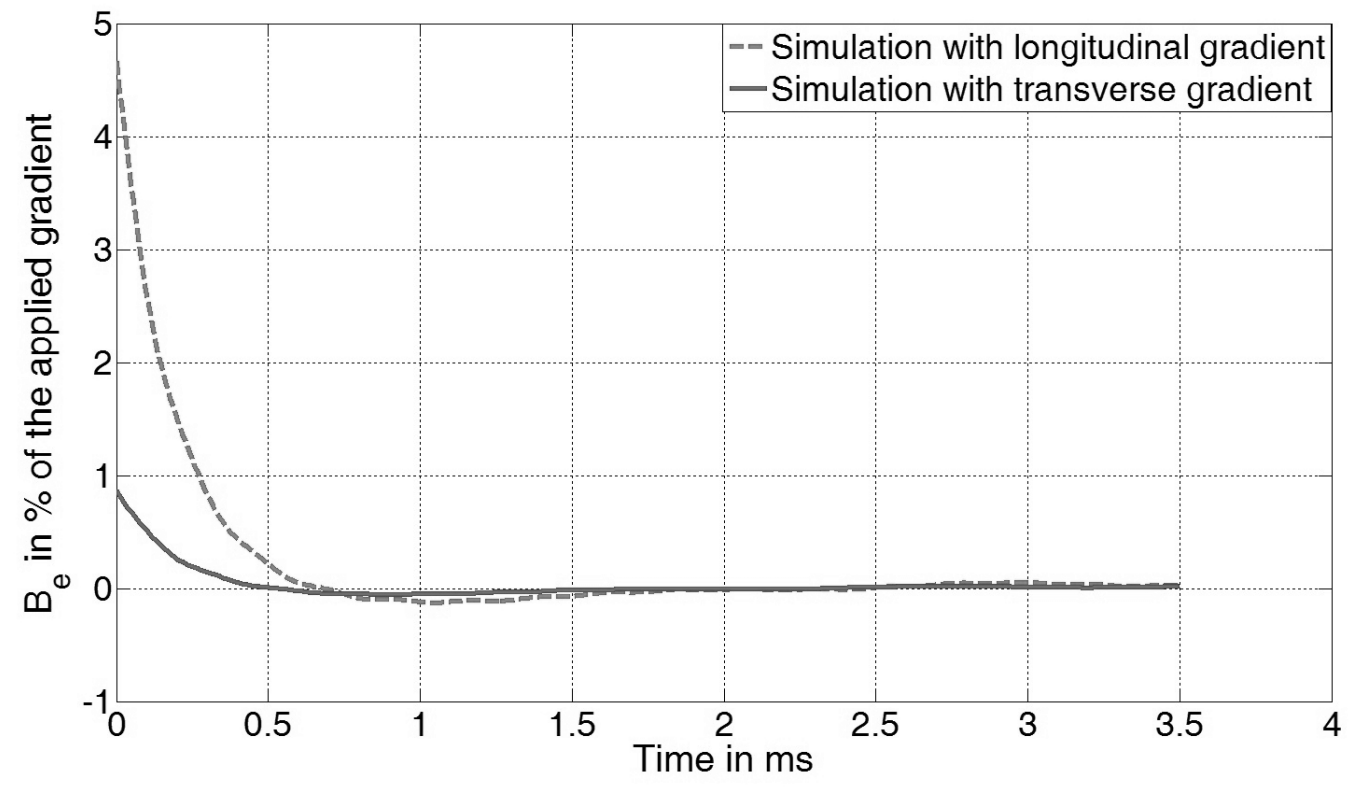

Figure 6

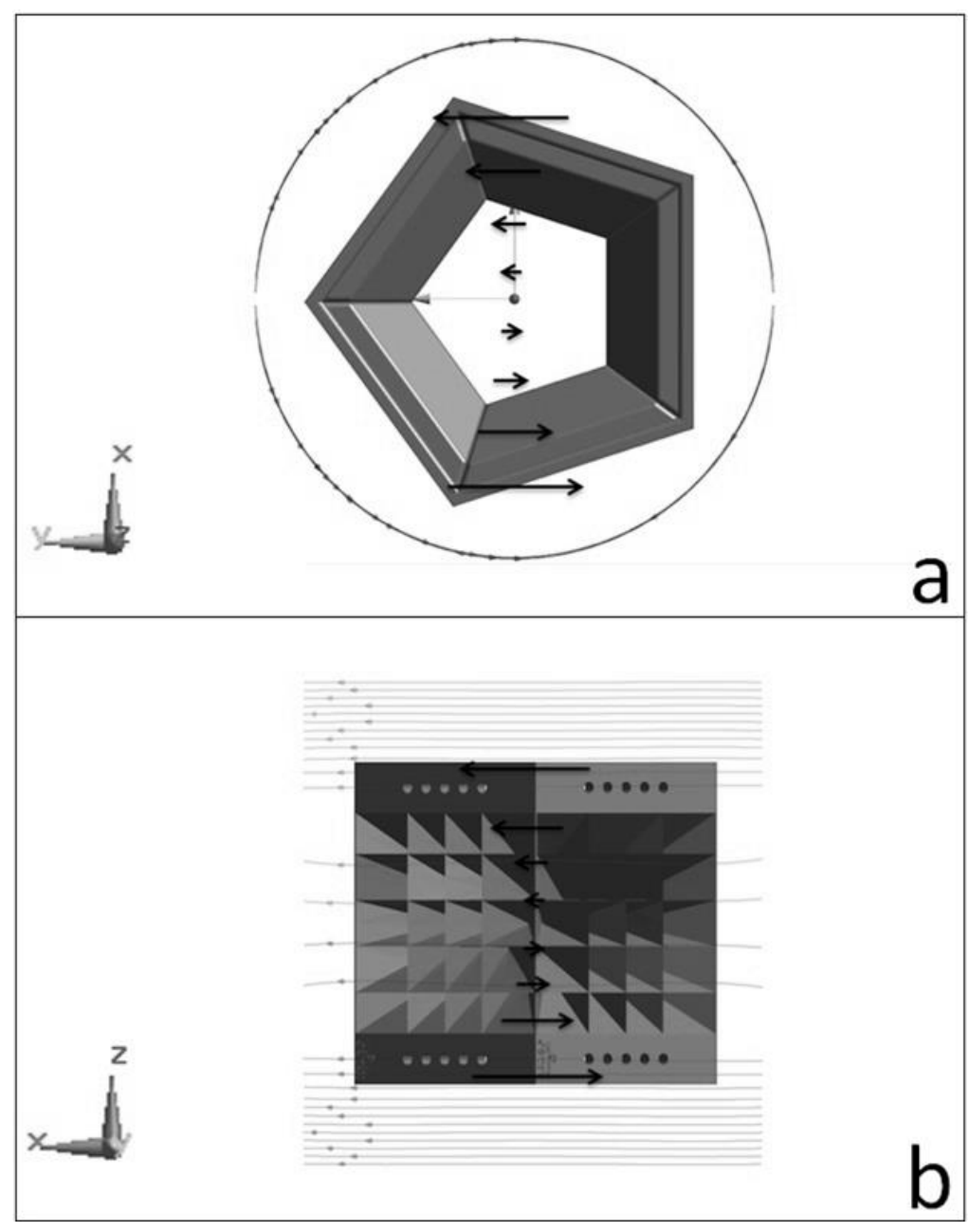

Figure 7 